\title{
SOLUTIONS OF A CLASS OF QUARTIC THUE INEQUALITIES
}

\author{
Bo He, Borka Jadrijević and Alain Togbé
}

ABa Teacher's College, China, University of Split, Croatia and Purdue University North Central, USA

Abstract. Let $c$ be a positive integer. In this paper, we use the method of Tzanakis to transform the quartic Thue inequality

$$
\left|x^{4}-4 x^{3} y-(2 c-2) x^{2} y^{2}+(4 c+4) x y^{3}-(2 c-1) y^{4}\right| \leq \max \left\{\frac{c}{4}, 4\right\}
$$

into systems of Pellian equations. Then we find all primitive solutions of this inequality using continued fractions.

\section{INTRODUCTION}

A Thue equation is a Diophantine equation of the form

$$
F(x, y)=k,
$$

where $F \in \mathbb{Z}[X, Y]$ is an irreducible binary form of degree $d \geq 3$ and $k$ is a non-zero rational integer; the unknowns $x$ and $y$ being rational integers. The name is given in honor of the Norwegian mathematician A. Thue ([16]) who proved that it has only finitely many solutions. Upper bounds for the solutions have been given using A. Baker's theory on linear forms in logarithms of algebraic numbers (see [1]). In fact, since E. Thomas ([15]) has solved the first parameterized family of Thue equations of positive discriminant, several families of parameterized Thue equations have been studied, many authors are able to solve cubic, quartic, quintic, sextic Thue equations. In 2004,

2000 Mathematics Subject Classification. 11D59, 11D09, 11D75, 11A55.

Key words and phrases. Thue inequalities, method of Tzanakis, continued fractions.

The first author is supported by Applied Basic Research Foundation of Sichuan Provincial Science and Technology Department (No.2009JY0091).

The second author was supported by the Ministry of Science, Education and Sports, Republic of Croatia, grant 037-0372781-2821.

The third author was supported in part by Purdue University North Central. 
Heuberger, Togbé, and Ziegler ([10]) solved the first octic family of Thue equations. In 2006, the third author solved a family of sextic Thue equations by means of Baker's method (see [17]).

In 1993, Tzanakis ([18]) considered Thue equations of the form $F(x, y)=$ $k$, where $F$ is a quartic form that corresponds to quartic field $\mathbb{K}$ which is the composite of two real quadratic fields. Tzanakis proved that solving the equation $F(x, y)=k$ consists in solving a system of Pellian equations. Therefore the method is called the Tzanakis method. The method was successfully applied by several authors not only for families of quartic Thue equations but also to families of quartic Thue inequalities. One can cite for examples $[6-9,11-13,21]$. One advantage of the method is that one can use the theory of continued fractions to determine small values of $k$ for which the equation $F(x, y)=k$ has a solution. Worley ([20]), Dujella ([5]), DujellaIbrahimpašić ([6]) have extended or generalized the classical results of Legendre and Fatou concerning Diophantine approximations of irrational numbers.

Let $c \geq 1$ be an integer. In this paper, we consider the Thue inequality

$$
\text { (1.1) }\left|x^{4}-4 x^{3} y-(2 c-2) x^{2} y^{2}+(4 c+4) x y^{3}-(2 c-1) y^{4}\right| \leq \max \left\{\frac{c}{4}, 4\right\} \text {, }
$$

or, equivalently, the Thue equations of the form

$$
x^{4}-4 x^{3} y-(2 c-2) x^{2} y^{2}+(4 c+4) x y^{3}-(2 c-1) y^{4}=m,
$$

where $|m| \leq \max \left\{\frac{c}{4}, 4\right\}$. The aim of the paper is to prove the following theorem.

THEOREM 1.1. Let $c, m$ be integers such that $c \geq 1$ and $|m| \leq \max \left\{\frac{c}{4}, 4\right\}$ and such that the Thue equation (1.2) has a primitive solution. Then

$$
m \in \begin{cases}\{1,-1,4,-4\}, & \text { if } c=1, \\ \{1,-3,4\}, & \text { if } c=2, \\ \{1,4\}, & \text { if } c \geq 3 .\end{cases}
$$

All primitive solutions are given as follows

1. If $c=2 n^{2}-2, n \in \mathbb{N}, n>1$,

$$
(x, y)= \begin{cases}( \pm 1,0), \pm(n+1, n), \pm(n-1, n) & \text { if } m=1 \\ \pm(1,1), \pm(2 n+1,1), \pm(2 n-1,-1) & \text { if } m=4\end{cases}
$$

2. If $c \neq 2 n^{2}-2, n \in \mathbb{N}, n>1$,

$$
(x, y)= \begin{cases}( \pm 1,0), & \text { if } m=1 \\ \pm(1,1), & \text { if } m=4\end{cases}
$$


Moreover, there are additional primitive solutions

$$
(x, y)=\left\{\begin{array}{ll}
(0, \pm 1), \pm(2,1), & \text { if } m=-1, \\
\pm(3,1), & \text { if } m=-4,
\end{array} \text { for } c=1\right.
$$

and

$$
(x, y)= \pm(2,1), \pm(0,1) \quad \text { if } m=-3, \text { for } c=2 .
$$

From the above theorem, one can easily deduce the following consequence.

Corollary 1.2. Let $c \geq 1$ be an integer. Then, for all $c \geq 1$, the Thue inequality (1.1) has primitive solutions of the form $(x, y)=( \pm 1,0), \pm(1,1)$. These solutions are the only primitive solutions if $c \neq 2 n^{2}-2, n \in \mathbb{N}, n>1$ and $c \neq 1,2$. The additional primitive solutions are given by:

i) $(x, y)= \pm(n+1, n), \pm(n-1, n), \pm(2 n+1,1), \pm(2 n-1,-1)$ for $c=2 n^{2}-2, n \in \mathbb{N}, n>1$

ii) $(x, y)= \pm(0,1), \pm(2,1)$ for $c=2$;

iii) $(x, y)=(0, \pm 1), \pm(2,1), \pm(3,1)$ for $c=1$.

First we will use Tzanakis method to transform the Thue equation (1.2) into the following system of Pellian equations

$$
\begin{aligned}
& c U^{2}-(c+4) V^{2}=-4 m, \\
& U^{2}-2(c+4) Z^{2}=m .
\end{aligned}
$$

This is done in the next section. Second, we recall some results due to Worley, Dujella, and Dujella-Jadrijević on continued fractions in Section 3. We use these results in order to find all possible values of $m$ for which system (1.3) and (1.4) has a solution. In the last two sections we solve equation (1.2) for all values of $m$ that are obtained in Section 3, using results of Cohn $([3,4])$ and Walsh ([19]).

\section{An APPlication of the Method of TZANAKis}

In this section, we recall the method of Tzanakis and use it to transform equation (1.2) into a system of Pellian equations. So let us consider the Thue equation

$$
f(x, y)=m
$$

where

(2.2) $f(x, y)=a_{0} x^{4}+4 a_{1} x^{3} y+6 a_{2} x^{2} y^{2}+4 a_{3} x y^{3}+a_{4} y^{4} \in \mathbb{Z}[x, y], a_{0}>0$.

We assign to this equation the cubic equation

$$
4 \rho^{3}-g_{2} \rho-g_{3}=0
$$


where

$$
g_{2}=a_{0} a_{4}-4 a_{1} a_{3}+3 a_{2}^{2} \in \frac{1}{12} \mathbb{Z}, \quad g_{3}=\left|\begin{array}{ccc}
a_{0} & a_{1} & a_{2} \\
a_{1} & a_{2} & a_{3} \\
a_{2} & a_{3} & a_{4}
\end{array}\right| \in \frac{1}{432} \mathbb{Z} .
$$

In 1993, Tzanakis [18] proved that a necessary condition to apply his method is that equation (2.3) has three rational roots $\rho_{1}, \rho_{2}, \rho_{3}$ that satisfy

$$
\frac{a_{1}^{2}}{a_{0}}-a_{2} \geq \max \left(\rho_{1}, \rho_{2}, \rho_{3}\right) .
$$

Let $H(x, y)$ and $G(x, y)$ be the quartic and sextic covariants of $f(x, y)$ respectively, i.e.

$$
\begin{gathered}
H(x, y)=-\frac{1}{144}\left|\begin{array}{cc}
\frac{\partial^{2} f}{\partial x^{2}} & \frac{\partial^{2} f}{\partial x \partial y} \\
\frac{\partial^{2} f}{\partial y \partial x} & \frac{\partial^{2} f}{\partial y^{2}}
\end{array}\right| \in \frac{1}{48} \mathbb{Z}[x, y], \\
G(x, y)=-\frac{1}{8}\left|\begin{array}{cc}
\frac{\partial f}{\partial x} & \frac{\partial f}{\partial y} \\
\frac{\partial H}{\partial x} & \frac{\partial H}{\partial y}
\end{array}\right| \in \frac{1}{96} \mathbb{Z}[x, y] .
\end{gathered}
$$

Then we have $4 H^{3}-g_{2} H f^{2}-g_{3} f^{3}=G^{2}$. Let us put

$$
H=\frac{1}{48} H_{0}, G=\frac{1}{96} G_{0}, \rho_{i}=\frac{1}{12} r_{i}, i=1,2,3,
$$

then

$$
H_{0}, G_{0} \in \mathbb{Z}[x, y], r_{i} \in \mathbb{Z}, i=1,2,3,
$$

and

$$
\left(H_{0}-4 r_{1} f\right)\left(H_{0}-4 r_{2} f\right)\left(H_{0}-4 r_{3} f\right)=3 G_{0}^{2} .
$$

There exist positive square-free integers $k_{1}, k_{2}, k_{3}$ and quadratic $G_{1}, G_{2}, G_{3} \in$ $\mathbb{Z}[x, y]$ such that

$$
H_{0}-4 r_{i} f=k_{i} G_{i}^{2}, \quad i=1,2,3
$$

and $k_{1} k_{2} k_{3}\left(G_{1} G_{2} G_{3}\right)^{2}=3 G_{0}^{2}$. If $(x, y) \in \mathbb{Z} \times \mathbb{Z}$ is a solution of (2.1), then we obtain

$$
\begin{aligned}
& k_{2} G_{2}^{2}-k_{1} G_{1}^{2}=4\left(r_{1}-r_{2}\right) m, \\
& k_{3} G_{3}^{2}-k_{1} G_{1}^{2}=4\left(r_{1}-r_{3}\right) m .
\end{aligned}
$$

Now we apply the reduction method to equation (1.2). In this particular case, we obtain

$$
\begin{gathered}
a_{0}=1, \quad a_{1}=-1, \quad a_{2}=-\frac{c-1}{3}, \quad a_{3}=c+1, \quad a_{4}=-2 c+1, \\
g_{2}=\frac{c^{2}+4 c+16}{3}, \quad g_{3}=\frac{c^{3}+6 c^{2}-24 c-64}{27} .
\end{gathered}
$$


One can see that equation (2.3) has three rational roots

$$
\rho_{1}=-\frac{1}{6} c+\frac{2}{3}, \quad \rho_{2}=-\frac{1}{6} c-\frac{4}{3}, \quad \rho_{3}=\frac{1}{3} c+\frac{2}{3} .
$$

Since

$$
\frac{a_{1}^{2}}{a_{0}}-a_{2}=\frac{1}{3} c+\frac{2}{3} \geq \max \left\{\rho_{1}, \rho_{2}, \rho_{3}\right\}
$$

so the reduction method can be applied to equation (1.2). We get

$$
\begin{aligned}
& H_{0}(x, y)-4 r_{1} F(x, y)=24 c\left(x^{2}-2 x y+3 y^{2}\right)^{2}, \\
& H_{0}(x, y)-4 r_{2} F(x, y)=24(c+4)\left(x^{2}-2 x y-y^{2}\right)^{2}, \\
& H_{0}(x, y)-4 r_{3} F(x, y)=48 c(c+4)\left(x y-y^{2}\right)^{2} .
\end{aligned}
$$

We take

$$
k_{1}=6 c, k_{2}=6(c+4), k_{3}=3 c(c+4),
$$

and

$$
G_{1}=2\left(x^{2}-2 x y+3 y^{2}\right), G_{2}=2\left(x^{2}-2 x y-y^{2}\right), G_{3}=4 y(x-y) .
$$

Let

$$
U=x^{2}-2 x y+3 y^{2}, \quad V=\left|x^{2}-2 x y-y^{2}\right|, \quad Z=|y(x-y)| .
$$

Then, by the method of Tzanakis, solving equation (1.2) reduces to solving the system of Pellian equations (1.3) and (1.4), where $|m| \leq \max \left\{\frac{c}{4}, 4\right\}$.

Assuming that $x$ and $y$ are relatively prime, from $(2.7)$ we see that $\operatorname{gcd}(U, V, Z)=1$. Since $U^{2}-V^{2}=8 Z^{2}$, we see $\operatorname{gcd}(U, V)=1$ or 2 .

\section{Continued fractions}

In this section, we will consider the connections between solutions of the equations (1.3) and continued fraction expansion of the corresponding quadratic irrational.

The simple continued fraction expansion of a quadratic irrational $\alpha=$ $\frac{a+\sqrt{d}}{b}$ is periodic. This expansion can be obtained using the following algorithm. Multiplying the numerator and the denominator by $b$, if necessary, we may assume that $b \mid\left(d-a^{2}\right)$. Let $s_{0}=a, t_{0}=b$ and

$$
a_{n}=\left\lfloor\frac{s_{n}+\sqrt{d}}{t_{n}}\right\rfloor, \quad s_{n+1}=a_{n} t_{n}-s_{n}, \quad t_{n+1}=\frac{d-s_{n+1}^{2}}{t_{n}}, \quad \text { for } n \geq 0 .
$$

If $\left(s_{j}, t_{j}\right)=\left(s_{k}, t_{k}\right)$ for $j<k$, then

$$
\alpha=\left[a_{0}, \ldots, a_{j-1}, \overline{a_{j}, \ldots, a_{k-1}}\right] .
$$


Applying this algorithm to quadratic irrational $\sqrt{(c+4) / c}$, we find

$$
\begin{gathered}
\sqrt{\frac{c+4}{c}}=[1 ; \bar{c}, 2], \\
\left(s_{0}, t_{0}\right)=(0, c),\left(s_{1}, t_{1}\right)=(c, 4), \\
\left(s_{2}, t_{2}\right)=(c, c),\left(s_{3}, t_{3}\right)=(c, 4),
\end{gathered}
$$

if $c$ is an even positive integer, and

$$
\begin{gathered}
\sqrt{\frac{c+4}{c}}=\left[1, \overline{\frac{c-1}{2}, 1,2 c+2,1, \frac{c-1}{2}, 2}\right], \\
\left(s_{0}, t_{0}\right)=(0, c),\left(s_{1}, t_{1}\right)=(c, 4), \\
\left(s_{2}, t_{2}\right)=(c-2,2 c-1),\left(s_{3}, t_{3}\right)=(c+1,1), \\
\left(s_{4}, t_{4}\right)=(c+1,2 c-1),\left(s_{5}, t_{5}\right)=(c-2,4), \\
\left(s_{6}, t_{6}\right)=(c, c),\left(s_{7}, t_{7}\right)=(c, 4),
\end{gathered}
$$

if $c>1$ is an odd positive integer. If $c=1$, we have

$$
\begin{gathered}
\sqrt{\frac{c+4}{c}}=\sqrt{5}=[2, \overline{4}], \\
\left(s_{0}, t_{0}\right)=(0,1),\left(s_{1}, t_{1}\right)=(2,1),\left(s_{2}, t_{2}\right)=(2,1) .
\end{gathered}
$$

Let $(U, V, Z)$ be integer solution of the system of equations (1.3) and (1.4). One can observe that if one of the integers $U, V, Z$ is equal to zero, then equations (1.3) and (1.4) imply that the two other integers are not equal to zero. Moreover, one can see that $U=0$ or $V=0$ is impossible, which follows immediately from $U^{2}-V^{2}=8 Z^{2}$.

Assume now that $(U, V, Z)$ is a nonnegative solution of the system (1.3) and (1.4). Then $\frac{U}{V}$ is a good rational approximation of $\sqrt{\frac{c+4}{c}}$. We find that

$$
\sqrt{\frac{c+4}{c}}+\frac{U}{V}>\sqrt{\frac{c+4}{c}}
$$

which implies

$$
\begin{aligned}
\left|\sqrt{\frac{c+4}{c}}-\frac{U}{V}\right| & =\left|\frac{c+4}{c}-\frac{U^{2}}{V^{2}}\right| \cdot\left|\sqrt{\frac{c+4}{c}}+\frac{V}{U}\right|^{-1} \\
& <\frac{4|m|}{c V^{2}} \cdot \sqrt{\frac{c}{c+4}} \leq \begin{cases}\frac{4 \cdot \frac{c}{4}}{c V^{2}} \cdot 1=\frac{1}{V^{2}}, & \text { if } c \geq 16, \\
\frac{16}{\sqrt{c(c+4)} V^{2}}, & \text { if } c \leq 15 .\end{cases}
\end{aligned}
$$

This implies that

$$
\left|\sqrt{\frac{c+4}{c}}-\frac{U}{V}\right|<\frac{M}{V^{2}},
$$


where $M=1$ if $c \geq 15, M=2$ if $7 \leq c \leq 14, M=3$ if $4 \leq c \leq 6, M=4$ if $c=3, M=5$ if $c=2$ and $M=8$ if $c=1$.

Let $p_{k} / q_{k}$ denote the $k^{\text {th }}$ convergent of $\alpha$. The following result of Worley ([20]) and Dujella ([5]) extends classical results of Legendre and Fatou concerning Diophantine approximations of the form $\left|\alpha-\frac{a}{b}\right|<\frac{1}{2 b^{2}}$ and $\left|\alpha-\frac{a}{b}\right|<\frac{1}{b^{2}}$.

Theorem 3.1 (Worley [20], Dujella [5]). Let $\alpha$ be a real number and a and $b$ coprime nonzero integers, satisfying the inequality

$$
\left|\alpha-\frac{a}{b}\right|<\frac{M}{b^{2}}
$$

where $M$ is a positive real number. Then $(a, b)=\left(r p_{k+1} \pm u p_{k}, r q_{k+1} \pm u q_{k}\right)$, for some $k \geq-1$ and nonnegative integers $r$ and $u$ such that $r u<2 M$.

Explicit version of Theorem 3.1 for $M=2$, was given by Worley ([20, Corollary, p. 206]). Recently, Dujella and Ibrahimpašić ([6, Propositions 2.1 and 2.2]) extended Worley's work and gave explicit and sharp versions of Theorem 3.1 for $M=3,4, \ldots, 12$.

We would like to apply Theorem 3.1 in order to determine all values of $m$ with $|m| \leq \max \left\{\frac{c}{4}, 4\right\}$ for which equation (1.3) has solutions. We need the following lemma (see Dujella and Jadrijević [8, Lemma 1]).

LEMMA 3.2. Let $\alpha \beta$ be a positive integer which is not a perfect square, and let $p_{k} / q_{k}$ denotes the $k$ th convergent of continued fraction expansion of $\sqrt{\frac{\alpha}{\beta}}$. Let the sequences $\left(s_{k}\right)$ and $\left(t_{k}\right)$ be defined by (3.1) for the quadratic irrational $\frac{\sqrt{\alpha \beta}}{\beta}$. Then

$$
\alpha\left(r q_{k+1}+u q_{k}\right)^{2}-\beta\left(r p_{k+1}+u p_{k}\right)^{2}=(-1)^{k}\left(u^{2} t_{k+1}+2 r u s_{k+2}-r^{2} t_{k+2}\right) .
$$

Since the period length of the continued fraction expansion of $\sqrt{\frac{c+4}{c}}$ is: equal to 2 if $c$ is even, equal to 6 if $c>1$ is odd and equal to 1 if $c=1$, according to Lemma 3.2, we have to consider only the fractions $\left(r p_{k+1}+\right.$ $\left.u p_{k}\right) /\left(r q_{k+1}+u q_{k}\right)$ for: $k=0,1$ if $c$ is even and $c=1$ and $k=0,1, \ldots, 5$ if $c>1$ is odd.

By checking all possibilities, it is now easy to prove the following result.

Proposition 3.3. Let $c$ and $m$ be integers such that $c \geq 1$ and $|m| \leq$ $\max \left\{\frac{c}{4}, 4\right\}$ and such that equation (1.3) has a solution in integers $U$ and $V$ 
with $\operatorname{gcd}(U, V)=1$ or 2 . Then

$$
m \in \begin{cases}\left\{1,4,-\frac{c}{4}\right\}, & \text { if } c \equiv 0 \quad(\bmod 4), c \geq 16, \\ \{1,4\}, & \text { if } c \neq 0 \quad(\bmod 4), c \geq 13 \text { and } c=10,9,6,5, \\ \{1,-3,4\}, & \text { if } c=12,2, \\ \{1,-2,4\}, & \text { if } c=11, \\ \{1,-2,3,4\}, & \text { if } c=8, \\ \{1,3,4\}, & \text { if } c=7, \\ \{1,-1,2,-2,4,-4\}, & \text { if } c=4, \\ \{1,2,-2,-3,4,-4\}, & \text { if } c=3, \\ \{1,-1,4,-4\}, & \text { if } c=1 .\end{cases}
$$

Furthermore, all solutions of this equation in relatively prime positive integers are given by $(U, V)=\left(p_{2 k}, q_{2 k}\right)$ if $m=1$ and $c \equiv 0(\bmod 4), c \geq 16$; $(U, V)=\left(p_{2 k+1}, q_{2 k+1}\right)$ if $m=-\frac{c}{4}$ and $c \equiv 0(\bmod 4) ;(U, V)=\left(p_{6 k}, q_{6 k}\right)$, $\left(p_{6 k+2}+p_{6 k+2}, q_{6 k+2}+q_{6 k+2}\right),\left(p_{6 k+4}, q_{6 k+2}\right)$ if $m=1$ and $c \equiv 1,3(\bmod 4)$, $c \geq 15$. While all solutions of this equation in integers with $\operatorname{gcd}(U, V)=2$ are given by $(U, V)=\left(2 p_{2 k}, 2 q_{2 k}\right)$ if $m=4$ and $c \equiv 0(\bmod 4), c \geq 16$; $(U, V)=\left(2 p_{6 k}, 2 q_{6 k}\right),\left(2 p_{6 k+2}+2 p_{6 k+2}, 2 q_{6 k+2}+2 q_{6 k+2}\right),\left(2 p_{6 k+4}, 2 q_{6 k+2}\right)$ if $m=4$ and $c \equiv 1,3(\bmod 4), c \geq 15$.

Here $p_{k} / q_{k}$ denotes the $k^{\text {th }}$ convergent of continued fraction expansion of $\sqrt{\frac{c+4}{c}}$ and $\left(p_{-1}, q_{-1}\right)=(1,0)$.

Now we will discuss the solvability in integers $U, V, Z$, with $\operatorname{gcd}(U, V)=1$ or 2, of the system of equations (1.3) and (1.4) where $m$ is one of the admissible values from Proposition 3.3.

Assume now that for $m=-\frac{c}{4}$ and $c \equiv 0(\bmod 4), c \geq 16$, the system (1.3) and (1.4) has a solution $(U, V, Z)$. Then, by Proposition 3.3, we have $U=p_{2 k+1}$ for an integer $k \geq 0$.

We will need the recursive relations for the convergents of $\sqrt{\frac{c+4}{c}}, c \equiv 0$ $(\bmod 4)$, with odd and even subscripts. Let $c=4 d, d \geq 1$. Then from

$$
\begin{aligned}
p_{0} & =1, p_{1}=2 d+1, \\
p_{2 k} & =2 p_{2 k-1}+p_{2 k-2}, \\
p_{2 k+1} & =2 d p_{2 k}+p_{2 k-1},
\end{aligned}
$$

it follows easily

$$
p_{2 k+1}=2(2 d+1) p_{2 k-1}-p_{2 k-3} .
$$

The following lemma can be proved easily by induction. 
Lemma 3.4. Let the sequences $\left(p_{m}\right)$ be defined by (3.3), (3.4), and (3.5). Then for all $l \geq 0$ we have

$$
\begin{aligned}
& p_{4 l+3} \equiv 1 \quad(\bmod 8 d+8), \\
& p_{4 l+1} \equiv 2 d+1 \quad(\bmod 8 d+8) .
\end{aligned}
$$

From equation (1.4) we have

$$
U^{2}+d \equiv 0 \quad(\bmod 8 d+8) .
$$

On the other hand, if $c=4 d$ and $d \geq 4$, from Lemma 3.4 and $U=p_{2 k+1}$, we have

$$
U^{2}+d \equiv 1+d \not \equiv 0 \quad(\bmod 8 d+8) \quad \text { if } \quad U=p_{4 l+3}
$$

or

$U^{2}+d \equiv(2 d+1)^{2}+d \equiv(d+1)(4 d+1) \not \equiv 0 \quad(\bmod 8 d+8) \quad$ if $U=p_{4 l+1}$. Hence, we have proved that for $m=-\frac{c}{4}$ and $c \equiv 0(\bmod 4), c \geq 16$ the system (1.3) and (1.4) has no solution. We will consider the remaining cases in the next two sections.

$$
\text { 4. CASES } m=1,4
$$

Let $c \geq 1$ and $m=1,4$. Let $x=X+Y$ and $y=Y$. Then equation (1.2) gives

$$
\left(2 Y^{2}\right)^{2}-(c+2)\left(2 Y^{2}\right) X^{2}+X^{4}=m .
$$

Furthermore, by (2.7), we have $(U, V, Z)=\left(X^{2}+2 Y^{2},\left|X^{2}-2 Y^{2}\right|,|X Y|\right)$.

We will apply the results of Cohn [3, Equation 1 and Equation 5] and Walsh [19, Theorem 2].

Theorem 4.1 (Cohn [3]). Let $K$ be an odd positive integer.

1. The only solutions to $x^{2}-K x y^{2}+y^{4}=1$ in non-negative integers $(x, y)$ are $(K, 1),(0,1),(1,0)$, unless either $K$ is a perfect square, in which case there is also the solution $(1, \sqrt{K})$;

2. The only solution in non-negative integers $x, y$ to the equation $x^{2}-$ $K x y^{2}+y^{4}=4$ is $(x, y)=(2,0)$.

Theorem 4.2 (Walsh [19]). Let $K$ be an even positive integer.

1. The only solutions to $x^{2}-K x y^{2}+y^{4}=1$ in non-negative integers $(x, y)$ are $(K, 1),(0,1),(1,0)$, unless either $K$ is a perfect square, in which case there are also the solutions $(1, \sqrt{K}),\left(K^{2}-\right.$ $1, \sqrt{K})$, or $K=338$ in which there are the solutions $(x, y)=$ $(114243,6214),(13051348805,6214)$

2. The only solution in non-negative integers $x, y$ to the equation $x^{2}-$ $K x y^{2}+y^{4}=4$ is $(x, y)=(2,0)$, unless $K=2 v^{2}$ for some integer $v$, in which case there are also the solutions $(2, \sqrt{2 K}),\left(2 K^{2}-2, \sqrt{2 K}\right)$. 
Let us begin with $m=1$ by applying the first part of the above theorems to equation (4.1).

CASE 1: For all $c \geq 1$, we obtain that possible solutions are given by $\left(2 Y^{2}, \pm X\right)=(c+2,1),(0,1),(1,0)$.

- The first solution gives us $c=2 n^{2}-2, X= \pm 1, Y= \pm n$ for some positive integer $n$. This implies $(U, V, Z)=\left(2 n^{2}+1,2 n^{2}-1, n\right)$ and the solutions of equation (1.2) are $(x, y)= \pm(n+1, n), \pm(n-1, n)$.

- The second solution gives us $X= \pm 1, Y=0$. This implies $(U, V, Z)=$ $(1,1,0)$, and the solutions are $(x, y)=( \pm 1,0)$.

- It is obvious to see that there is no solution corresponding to $\left(2 Y^{2}, \pm X\right)=(1,0)$.

CASE 2: If $c+2=t^{2}$, for some positive integer $t$, then possible additional solutions are given by $\left(2 Y^{2}, \pm X\right)=(1, t)$ for all $c \geq 1$ and $\left(2 Y^{2}, \pm X\right)=$ $\left(t^{4}-1, t\right)$ when $c$ is an even integer.

- Using Cohn's result [4], one can see that the equation $2 Y^{2}=t^{4}-1$ has only the integer solutions $(Y, t)=(0, \pm 1)$. This implies $c=-1$ which gives us a contradiction.

- The second eventuality $\left(2 Y^{2}, \pm X\right)=(1, t)$ is also impossible.

CASE 3: If $c+2=338$, i.e., $c=340$, then there is no additional solution because $2 Y^{2} \notin\{114243,13051348805\}$.

Now we only apply the second part of Theorem 4.1 and Theorem 4.2 to deal with $m=4$.

CASE 4: For all $c \geq 1$ possible solutions are given by $\left(2 Y^{2}, \pm X\right)=(2,0)$. This implies $(X, Y)=(0, \pm 1)$ and $(U, V, Z)=(2,2,0)$. Therefrom we obtain the solutions $(x, y)= \pm(1,1)$.

CASE 5: If $K=2 n^{2}$, i.e. $c=2 n^{2}-2$ with $n>1$, then we have two additional possibilities.

- First, we take $\left(2 Y^{2}, \pm X\right)=(2,2 n)$. Therefore, we get $Y= \pm 1$. It follows that $(U, V, Z)=\left(2 n^{2}+2,2 n^{2}-2,2 n\right)$, and gives the solutions $(x, y)=$ $\pm(2 n+1,1), \pm(2 n-1)$.

- Second, we take $\left(2 Y^{2}, \pm X\right)=\left(2\left(2 n^{2}\right)^{2}-2,2 n\right)$ and we get $Y^{2}=\left(2 n^{2}\right)^{2}-$ 1. This is impossible.

Therefore, all solutions are found when $m=1,4$.

REMARK 4.3. It is easy to see that when $m=4$ and $X=2 X_{1}$, then from (4.1), we get

$$
\left(2 X_{1}^{2}\right)^{2}-(c+2)\left(2 X_{1}^{2}\right) Y^{2}+Y^{4}=1 .
$$

Conversely, one can see that any solution of equation (4.1) of the form $(X, Y)=(a, b)$ for $m=1$ gives a solution of the form $(X, Y)=(2 b, a)$ for $m=4$. 


\section{CASES $m \neq 1,4$}

In this section, we study the remaining cases when $m \neq 1,4$. By (1.3) and (1.4), we get

$$
V^{2}-2 c Z^{2}=m .
$$

So if $c=2$ and $m=-3$ then from equation (5.1) we obtain

$$
V^{2}-4 Z^{2}=(V-2 Z)(V+2 Z)=-3 \text {. }
$$

This implies $V= \pm 1, Z= \pm 1$. Then, from (2.7), we obtain $(x, y)= \pm(2,1)$ or $(x, y)= \pm(0,1)$.

Now let us rewrite equation (4.1) to

$$
A^{2}-\left(\delta^{2}-4\right) B^{2}=m,
$$

where $\delta=c+2 \geq 3, A=X^{2}-(c+2) Y^{2}=(x-y)^{2}-(c+2) y^{2}, B=Y^{2}=y^{2}$. Since the period of continued fraction expansion of $\sqrt{\delta^{2}-4}$ is even if $\delta \geq 4$, there is no solution if $m=-1,-4$ and $c \geq 2$. If $\delta=3$, i.e $c=1$, the period is odd which implies that equation (5.2) has solutions with $m=-1$ and $m=-4$.

It remains to consider the cases:

- $c=1$ and $m=-1$ or $m=-4$;

- $c=3$ and $m=2$ or $m=-2$ or $m=-3$;

- $c=7$ and $m=-3$;

- $c=8$ and $m=-2$ or $m=3$;

- $c=11$ and $m=-2$;

- $c=12$ and $m=-3$.

We solve the remaining cases as follows.

CASE $c=1$. When $m=-1$, equation (1.2) was solved by Tzanakis [18, p. 275]. For $m=-4$, we used Thuesolver of KASH ([14]). So we get

$$
(x, y)= \begin{cases}(0, \pm 1), \pm(2,1), & \text { if } m=-1 \\ \pm(3,1), & \text { if } m=-4 .\end{cases}
$$

CASE $c=3$. Equation (5.2) becomes $A^{2}-21 B^{2}=m$. If $m=2$, it implies $A^{2} \equiv 2(\bmod 3)$. This is impossible. If $m=-2$, we obtain $A^{2} \equiv-2 \equiv 5$ $(\bmod 7)$. So there is no solution. If $m=3$, then $A^{2} \equiv 3(\bmod 7)$, but 3 is not a square modulo 7 . Therefore, we have no solution $(A, B)$ when $c=3$.

CASE $c=7, m=-3$. In this case, equation (5.2) has the form $A^{2}-$ $77 B^{2}=-3$. By taking modulo 11 , we obtain $A^{2} \equiv-3(\bmod 11)$. Using the Legendre symbol, we get $(-3 / 11)=(8 / 11)=(2 / 11)=-1$. There is no solution $(A, B)$.

CASE $c=8$. Equation (5.2) becomes $A^{2}-96 B^{2}=m$. If $m=-2$, we get $A^{2} \equiv-2 \equiv 2(\bmod 4)$ which is impossible. If $m=3$, similarly, $A^{2} \equiv 3$ $(\bmod 4)$ gives us a contradiction. 
CASE $c=11, m=-2$. Then we have $A^{2}-165 B^{2}=-2$. We get that $A^{2} \equiv-2(\bmod 5)$. This is impossible.

CASE $c=12, m=-3$. From equation (5.2), we deduce $A^{2}-192 B^{2}=-3$. Then $A^{2} \equiv-3 \equiv 5(\bmod 8)$ which is also impossible.

This completes the proof of Theorem 1.1.

ACKNOWLEDGEMENTS.

The authors would like to thank Professor Andrej Dujella for his many helpful comments and suggestions.

\section{REFERENCES}

[1] A. Baker, Contribution to the theory of Diophantine equations. I. On the representation of integers by binary forms, Philos. Trans. Roy. Soc. London Ser. A 263 (1968), 173-191.

[2] A. Baker and H. Davenport, The equations $3 x^{2}-2=y^{2}$ and $8 x^{2}-7=z^{2}$, Quart. J. Math. Oxford 20 (1969), 129-137.

[3] J. H. E. Cohn, Twelve Diophantine equations, Arch. Math. 65 (1995), 130-133.

[4] J. H. E. Cohn, The Diophantine equation $x^{4}-D y^{2}=1$ II, Acta Arith. 78 (1997), 401-403.

[5] A. Dujella, Continued fractions and RSA with small secret exponent, Tatra Mt. Math. Publ. 29 (2004), 101-112.

[6] A. Dujella and B. Ibrahimpašić, On Worley's theorem in Diophantine approximations, Ann. Math. Inform. 35 (2008), 61-73.

[7] A. Dujella and B. Jadrijević, A parametric family of quartic Thue equations, Acta Arith. 101 (2002), 159-169.

[8] A. Dujella and B. Jadrijević, A family of quartic Thue inequations, Acta Arith. 111 (2004), 61-76.

[9] A. Dujella, B. Ibrahimpašić and B. Jadrijević, Solving a family of quartic Thue inequalities using continued fractions, preprint.

[10] C. Heuberger, A. Togbé and V. Ziegler, Automatic solutions of families of Thue equations and an example of degree 8, J. Symbolic Comput. 38 (2004), 1145-1163.

[11] B. Ibrahimpašić, A parametric family of quartic Thue inequalities, preprint.

[12] B. Jadrijević, A system of Pellian equations and related two-parametric family of quartic Thue equations, Rocky Mountain J. Math. 35 (2005), 547-572.

[13] B. Jadrijević and V. Ziegler, A system of relative Pellian equations and a family of relative Thue inequations, International Journal of Number theory, 2 (2006), 569-590.

[14] M. Daberkow, C. Fieker, J. Kluners, M. E. Pohst, K, Roegner, and K. Wildanger, Kant V4, J. Symbolic Comput. 24 (1997) 267-283.

[15] E. Thomas, Complete solutions to a famaly of cubic Diophantine equations, J. Number Theory 34 (1990), 235-250.

[16] A. Thue, Über Annäherungswerte algebraischer Zahlen, J. Reine Angew. Math., 135 (1909), 284-305.

[17] A. Togbé, A parametric family of sextic Thue equations Acta Arith., 125 (2006), 347-361.

[18] N. Tzanakis, Explicit solution of a class of Quartic Thue Equations, Acta. Arith. 64 (1993), 271-283.

[19] P. G. Walsh, A note on a theorem of Ljunggren and the Diophantine equations $x^{2}-$ $k x y^{2}+y^{4}=1,4$, Arch. Math. 73 (1999), 119-125.

[20] R. T. Worley, Estimating $|\alpha-p / q|$, J. Austral. Math. Soc. Ser. A 31 (1981), 202-206. 
[21] V. Ziegler, On the certain family of quartic equations with three parameters, Glas. Mat. Ser. III 41(61) (2006), 9-30.

Bo $\mathrm{He}$

Department of Mathematics

ABa Teacher's College

Wenchuan, Sichuan, 623000

P. R. China

E-mail: bhe@live.cn

B. Jadrijević

Faculty of Science

Department of Mathematics

University of Split

Teslina 12, 21000 Split

Croatia

E-mail: borka@pmfst.hr

A. Togbé

Mathematics Department

Purdue University North Central

1401 S, U.S. 421, Westville IN 46391

USA

E-mail: atogbe@pnc.edu

Received: 4.11.2008. 